\title{
Pobreza y remesas internacionales Sur-Sur en Paraguay
}

\author{
Pablo Sebastián Gómez \\ Eduardo Bologna ${ }^{* *}$
}

La discusión sobre migración y desarrollo está fuertemente vinculada al papel de las remesas internacionales. Sin embargo, no existe consenso sobre los efectos que ellas provocan en las economías de origen y los hogares receptores. El objetivo de este artículo es analizar el efecto de la recepción de remesas regionales en el nivel y la incidencia de la pobreza de los hogares paraguayos. Para ello se utilizan microdatos de la Encuesta Permanente de Hogares de Paraguay de 2009, de la Dirección General de Estadística, Encuestas y Censos (DGEEC). Se consideran remesas regionales las provenientes de Argentina y Brasil. Se utiliza el método de análisis de la puntuación de la propensión (propensity score analysis) para construir grupos comparables y aislar el efecto de la recepción de remesas de otras variables que inciden sobre la condición de pobreza de los hogares. Se sugiere que las remesas tendrían un efecto positivo en la incidencia de la pobreza y el alivio de la pobreza extrema. Sin embargo, el resultado no es completamente concluyente, ya que la pobreza no extrema afecta en mayor medida a los hogares receptores de remesas.

Palabras clave: Remesas. Pobreza. Inferencia causal. Paraguay.

\footnotetext{
* Consejo Nacional de Investigaciones Científicas y Técnicas (CONICET), Universidad Nacional de Córdoba, Córdoba, Argentina (pablosgomezpsg@yahoo.com.ar).

** Centro de Estudios Avanzados, Universidad Nacional de Córdoba, Córdoba, Argentina (ebologna@gmail.com).
} 


\section{Introducción}

Las remesas internacionales constituyen uno de los tópicos centrales de la relación entre migración y desarrollo. Sin embargo, no existe consenso sobre los efectos que ellas provocan en las economías de origen y los hogares receptores. Para algunos académicos que han desarrollado su trabajo desde el paradigma neoclásico, la emigración es positiva para las áreas de origen porque provee una válvula de escape a la pobreza y el desempleo y genera un flujo de remesas (TAYLOR; DYER, 2009; TAYLOR, 1999). Otros académicos, fundamentalmente vinculados a un enfoque estructuralista, señalan los efectos negativos para las áreas de origen, entre ellos, el drenaje de población hacia las áreas receptoras; la creciente dependencia de las remesas; su canalización hacia bienes de consumo y no de inversión, lo que contribuye a la diferenciación social y económica; la inflación de los precios de la tierra y su concentración en unos pocos propietarios (CANALES, 2008; MÁRQUEZ, 2010; WISE; MÁRQUEZ, 2007). Gran parte de estos debates ha estado centrada en procesos migratorios Sur-Norte, por lo que en este artículo se pretende aportar evidencia empírica sobre lo que ocurre en un sistema migratorio Sur-Sur de relevancia para América Latina.

El patrón más ampliamente estudiado de las migraciones internacionales es el que gruesamente puede denominarse "Sur-Norte", intentando referirse con esta expresión simplificada a desplazamientos que se dirigen desde áreas con escaso desarrollo hacia las más atractivas por sus posibilidades laborales, sus expectativas de ingreso, entre otras características. Es difícil estimar cuantitativamente el volumen de las migraciones entre estas dos mega-regiones, ya que el recorte no es unívoco, puesto que diversos organismos las clasifican de manera diferente, teniendo en cuenta el ingreso per cápita o el valor del Índice de Desarrollo Humano (IDH).

Ratha y Shaw (2007) estiman que casi la mitad de los migrantes provenientes de los países en desarrollo residen en otros países de similar nivel, es decir que la migración entre países del Sur es casi tan grande como la que se dirige al Norte global. No obstante, no hay una definición unívoca del "Sur", una noción que incluso no está relacionada con la posición geográfica (BAKEWELL, 2009), por lo que el interés de una clasificación así de amplia no es tanto de orden geográfico, sino que reside en el hecho que permite ilustrar la diferencia en la composición y las motivaciones de los desplazamientos que suceden:

- entre países separados por una amplia brecha de desarrollo económico, hacia los que la migración implica recorrer grandes distancias, sortear barreras legales rígidas y a menudo superar diferencias de idioma;

- entre países con diferencias más leves en su desarrollo económico, que comparten una posición periférica en el mundo, cuyas fronteras son permeables y que reconocen gradaciones dentro de ellos desde áreas fronterizas poco diferenciadas internacionalmente hasta grandes ciudades.

Si los determinantes económicos son centrales al momento de explicar los primeros, lo son menos cuando se discuten los flujos intrarregionales. En estos últimos casos, las redes horizontales (de ayuda mutua) y verticales (de contratación o tráfico), así como la 
reunificación familiar, son condicionantes de mayor peso explicativo que los desequilibrios en la distribución de la demanda de mano de obra. Por lo tanto, las migraciones llamadas "Sur-Sur" tienen especificidades que requieren un estudio diferenciado.

Argentina ha constituido un polo histórico de los flujos Sur-Sur, aceptando la denominación para aquellos que suceden dentro de las regiones periféricas. Este país ha recibido migrantes de Paraguay, Bolivia, Perú y Chile principalmente (MARSHALL; ORLANSKY, 1983). En el caso específico de Paraguay, la economía doméstica de los hogares receptores está afectada fuertemente por las remesas y existen dinámicas de autoselección que conectan ciertos hogares con espacios del Norte (España y Estados Unidos) y otros con espacios del Sur (Argentina y Brasil) (GÓMEZ, 2013). Efectivamente, según el origen del flujo monetario es posible establecer dos macrosistemas migratorios en Paraguay: uno vinculado al Norte y otro al Sur. Dos características son relevantes en este sentido: a) considerando a la población en general, los hogares receptores no son los de menores ingresos, y b) aquellos que reciben remesas de Estados Unidos o España tienen mejor posición socioeconómica que aquellos que las reciben de Argentina y Brasil (GÓMEZ, 2013). Estas dinámicas diferenciales también implican especificidades de las características sociodemográficas de los hogares y de las modalidades de envío, recepción y uso de las remesas internacionales (GÓMEZ, en prensa).

El objetivo de este trabajo es analizar el efecto de las remesas internacionales provenientes de Argentina y Brasil en los niveles de pobreza de los hogares paraguayos. El artículo está organizado de la siguiente manera: en la primera sección se aborda la importancia de las remesas para la economía paraguaya; en la segunda se exponen los antecedentes relativos a la relación entre las remesas y la pobreza en las áreas de origen. La tercera sección está destinada a presentar la fuente de datos utilizada y las estadísticas descriptivas sobre la pobreza y las remesas en Paraguay. En la cuarta parte se expone y aplica la metodología propensity score analysis para evaluar el efecto de las remesas internacionales en la pobreza. Se utiliza para ello el paquete Matchlt (HO et al., 2007, 2011) del software R (THE R CORE TEAM, 2014). En la quinta sección se discuten los resultados obtenidos y se presentan las conclusiones.

\section{Evolución y características de las remesas en Paraguay}

La Argentina es el principal país de origen de las remesas que recibe Paraguay provenientes del Sur global, constituyendo un corredor Sur-Sur de relevancia para la región. La historia de la migración paraguaya hacia la Argentina es de larga data (AYALA, 1941; RIVAROLA, 1980), y hay registros de 3.300 paraguayos residiendo en ese país ya en el censo de 1869. En el relevamiento censal de 1895 el registro alcanzó las 14.500 personas, y en el de 1914 el volumen casi duplicó ese número (BROWNING; GILLESPIE, 1979). Según estimaciones de Browning y Gillespie (1979), hacia el final de la Segunda Guerra Mundial (1947) los paraguayos viviendo en Argentina eran 93.000, y en 1960, 155.000.

Según el censo de 2001 esta cifra se duplicó con creces, alcanzando las 325.046 personas, lo que representaba el $0,9 \%$ de la población total y el 21,2\% de la población 
extranjera residente en ese país. De acuerdo a los datos del relevamiento de 2010, los paraguayos que viven en Argentina han crecido hasta las 550.713 personas, constituyen el 1,4\% de la población total y el 30,5\% de la población extranjera (INDEC, 2012). La emigración hacia Brasil, aunque mucho menor, también se inició inmediatamente después de la Guerra de la Triple Alianza (1864-1870). Los paraguayos emigrados se ubicaron primeramente en las zonas rurales sobre todo, en el estado de Mato Grosso; a partir de 1950 los destinos de esta emigración se diversificaron y se dirigieron hacia las grandes metrópolis, en especial São Paulo (PALAU; FISCHER; PEREZ, 1997). Según los datos de los dos últimos censos se pasó de 28.822 personas en la ronda del 2000 a 40.000 en la del 2010 (OIM, 2011).

Según estimaciones del Banco Mundial, en Paraguay las remesas internacionales han experimentado un incremento importante, fundamentalmente en la década de 1990. En el Gráfico 1 se puede apreciar la evolución de las remesas internacionales y su comparación con el flujo de inversión extranjera directa. Como se observa, en la década de 1990 se produjo un crecimiento de magnitud hasta 1995 que luego se revirtió hasta 2002, momento en que comenzó a crecer en forma sostenida.

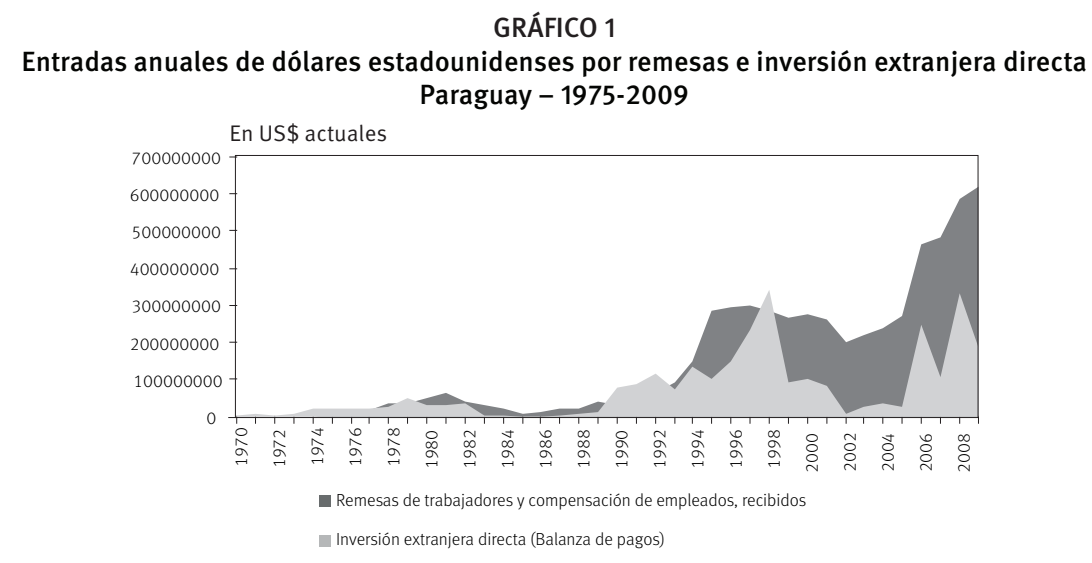

Fuente: World Development Indicators (WDI), World Bank (2013).

La importancia cuantitativa, así como la sostenibilidad en el tiempo de las remesas que reciben algunos hogares de Paraguay, sugieren la necesidad de ampliar los estudios de modo que contribuyan a dar cuenta de las consecuencias que estos ingresos tienen para los hogares que los reciben.

En la Tabla 1 se presentan diferentes indicadores de migración y remesas. Según esta información, más del 12\% de los hogares paraguayos recibe remesas y más del 10\% tiene miembros que han emigrado al exterior en los últimos 5 años. En cuanto a la procedencia de las remesas, se observan dos países principales, Argentina (63,2\%) y España (29,8\%), seguidos muy por debajo por Brasil y Estados Unidos, que no representan una magnitud de importancia.

Parrado y Cerrutti (2003) señalan que el flujo migratorio paraguayo más relevante, que se dirige hacia Argentina, se relaciona con las características individuales de los migrantes, 
la extensión de las redes sociales y la experiencia, pero también con los cambios en las condiciones macroeconómicas. Los resultados de su estudio muestran que este flujo migratorio es muy sensible a las fluctuaciones de las condiciones macroeconómicas, particularmente a los diferenciales de ingresos y los cambios en la valuación del peso argentino.

TABLA 1

Hogares vinculados a la migración y las remesas

Paraguay - 2009

\begin{tabular}{|c|c|}
\hline Tipo de hogar & Porcentaje sobre el total de hogares paraguayos \\
\hline Hogares receptores de remesas & 12,2 \\
\hline Hogares con miembros en el exterior (últimos 5 años) & 10,7 \\
\hline \multicolumn{2}{|c|}{ Procedencia de las remesas en hogares receptores } \\
\hline País de origen & Porcentaje \\
\hline Argentina & 63,17 \\
\hline España & 29,83 \\
\hline Brasil & 2,39 \\
\hline Estados Unidos & 2,03 \\
\hline Otro & 2,58 \\
\hline
\end{tabular}

Fuente: Encuesta Permanente de Hogares, 2009 (DGEEC, 2009a).

\section{Remesas y pobreza}

\section{Antecedentes}

La mayoría de los estudios empíricos sobre el tema señalan que las remesas provenientes de la migración internacional reducen la pobreza en los países receptores (ACOSTA et al., 2008; ADAMS; PAGE, 2005; ADAMS, 2006a, 2006b, 2011; HOBBS; JAMESON, 2012; TAYLOR; MORA; ADAMS, 2005; YANG; MARTÍNEZ, 2006). Sin embargo, en algunas circunstancias se encontró que el efecto del ingreso proveniente de las remesas en la reducción de la pobreza es sumamente pequeño y que depende del porcentaje de los hogares receptores que pertenecen a los quintiles de ingresos inferiores, en los que los efectos serían mayores (ACOSTA; FAJNZYLBER; LÓPEZ, 2007).

Se han encontrado efectos positivos de las remesas en la pobreza y la salud, y efectos negativos en la participación laboral, la educación y el crecimiento económico (ADAMS, 2011). Para Adams (2011), la importancia de examinar el impacto de las remesas en la pobreza o la desigualdad radica en que en la mayoría de las situaciones estas representan entre un $30 \%$ y un $40 \%$ del ingreso total de los hogares receptores, según los hallazgos realizados en varios contextos migratorios, por ejemplo, México (MASSEY; ALARCON; DURAND, 1990), República Dominicana y Guatemala (ITZIGSOHN, 1995).

En uno de los trabajos quizás más amplios sobre el tema, Adams y Page (2005) utilizan encuestas de hogares de 71 países para analizar el impacto de las remesas en la pobreza. Los resultados indican que un aumento del $10 \%$ en la proporción de migrantes internacionales de un país dará lugar a una disminución del 2,1\% en la proporción de personas que viven 
con menos de US \$1,00 por persona por día; de manera similar, un aumento del 10\% de las remesas internacionales per cápita conducirá a una disminución del 3,5\% de la proporción de personas viviendo en la pobreza.

Taylor, Mora y Adams (2005) concluyen también que en México la migración internacional y las remesas reducen la pobreza. Estos autores utilizan datos nacionales y regionales de encuestas del país para concluir que las remesas internacionales incrementan levemente la desigualdad entre los ingresos regionales y reducen la pobreza. Según los autores, los resultados refuerzan los argumentos de Stark, Taylor y Yitzhaki (1986), que postulan que la expansión de la migración tiene inicialmente efectos que acentúan las desigualdades en la distribución del ingreso rural, pero posteriormente, cuando la población accede en mayor medida a la migración, los efectos de las remesas en el ingreso rural se tornan más equitativos (o menos desiguales). Según Taylor, Mora y Adams (2005), esto podría explicar las inconsistencias del efecto estimado de las remesas en la desigualdad de los ingresos, ya que se utilizan datos de economías con diferentes niveles de integración de los migrantes en el mercado laboral. Además de estos efectos positivos sobre la desigualdad, las remesas internacionales reducen la pobreza, mucho más aún que las remesas internas. Este efecto es mayor cuando los migrantes están más integrados en las economías de destino. La dimensión temporal, según estos autores, es lo que explica gran parte de los resultados dispares en el estudio del efecto de las remesas en la pobreza.

Del mismo modo, Adams (2006a, 2006b) concluye que las remesas disminuyen la pobreza a partir de sus estudios sobre Guatemala y Ghana. El autor utiliza encuestas representativas de hogares y estima el ingreso contrafáctico de los hogares con y sin migrantes utilizando estimaciones econométricas para predecir el ingreso con y sin remesas. Después de controlar por la causalidad inversa y el sesgo de selección, encuentra que las remesas internas e internacionales reducen los niveles, la profundidad y la severidad de la pobreza en Ghana. Sin embargo, la magnitud de la reducción depende del tipo de remesas recibidas (internas o internacionales) y de la forma en que se mida la pobreza. Se reportan resultados similares para el caso de Guatemala.

Acosta et al. (2008) examinan el impacto de las remesas en 10 países de América Latina. Los autores construyen un escenario contrafactual comparando los niveles observados de pobreza con los que se hubieran registrado en una situación sin migración ni remesas en los hogares, a través del método de corrección propuesto por Heckman (1979). Los autores concluyen que la migración y las remesas tienen efectos estadísticamente significativos en la reducción de la pobreza, y que esta incidencia se produce básicamente mediante el incremento del ingreso per cápita en los países receptores de remesas. Los efectos en la pobreza varían según los diferentes países de América Latina: en aquellos en los que los hogares receptores se concentran en los estratos más bajos de la distribución del ingreso, como Ecuador, El Salvador, Guatemala, México y Paraguay, se advierte una mayor reducción de la pobreza atribuible a las remesas. El hecho de que los hogares receptores se encuentren entre los estratos socioeconómicos más desfavorecidos obedece a los bajos costos de la 
migración en relación con el ingreso y a la relativa proximidad de los principales países de destino, como en los casos de México y Paraguay.

Hobbs y Jameson (2012) examinan el impacto de las remesas en la pobreza en Nicaragua. Los autores utilizan la corrección propuesta por Heckman (1979) para predecir el escenario contrafáctico del consumo de los hogares según el supuesto de "no-migración”. Encuentran que la migración hacia Costa Rica provoca un incremento del consumo per cápita de los hogares de los sectores socioeconómicos más bajos, mientras que la migración hacia los Estados Unidos produce un aumento del consumo per cápita de aquellos ubicados en los quintiles de ingresos medios.

La mayoría de estos trabajos fueron realizados con una perspectiva económica, utilizando diferentes variantes de instrumentos econométricos y tomando en consideración una visión restringida y acotada de la definición de las remesas. Sin embargo, diversos autores señalan la necesidad de trascender esta definición meramente estadística (CANALES, 2008; MÁRQUEZ, 2010; WISE; MÁRQUEZ, 2007). Para ellos, las remesas internacionales no son solo dinero que los migrantes envían a sus familiares, son recursos salariales ${ }^{1}$ destinados a cubrir la subsistencia de los dependientes. Su función principal es la de reemplazar el salario, por lo que es un error teórico atribuirle propósitos de política pública (MÁRQUEZ, 2010).

Según la revisión bibliográfica realizada para este artículo, los trabajos que han utilizado el enfoque de la puntuación de la propensión para evaluar específicamente el impacto de las remesas en la pobreza son los de Esquivel y Huerta Pineda (2007) en México, Sharma (2011) en Sri Lanka, Jiménez-Soto y Brown (2012) en Tonga, Chukwuone et al. (2012) en Nigeria y López-Videla y Machuca (2014) en Bolivia.

\section{La medición del efecto de las remesas}

Para Jiménez-Soto y Brown (2012), gran parte de la discusión en el estudio del impacto de las remesas en la pobreza es metodológica, y se concentra en la forma de medir ese efecto. La importancia de la cuestión metodológica se explica porque la medición presenta dos problemas ampliamente conocidos:

- las remesas no pueden ser tratadas simplemente como una variable exógena al ingreso de los hogares receptores, ya que de esa forma se ignora lo que los migrantes podrían haber ganado si la emigración no hubiera ocurrido;

- no puede asumirse que los hogares receptores de remesas sean una muestra aleatoria del total de los hogares. Por el contrario, la pertenencia al grupo de los receptores es el resultado de un complejo conjunto de variables de nivel individual, del hogar y de las comunidades.

Esto ha dado origen a numerosos métodos para estimar el impacto de las remesas en la pobreza cuando se cuenta con datos de encuestas. Jiménez-Soto y Brown (2012) proponen una clasificación sencilla de estos métodos:

\footnotetext{
${ }^{1}$ En el caso de Paraguay estos recursos salariales son generados en condiciones específicas de inserción laboral. Véanse Bruno (2012), Maguid y Bruno (2010) y Cerrutti y Parrado (2007).
} 
- aquellos que apelan a modelos que utilizan variables instrumentales para estimar las tasas de pobreza observadas;

- aquellos que comparan las tasas de pobreza de los hogares con y sin remesas estimando el ingreso (contrafáctico) de los hogares si no las recibieran.

El primero en utilizar el abordaje contrafáctico en la literatura sobre la migración y las remesas fue Adams (1989), y el procedimiento fue refinado por Barham y Boucher (1998).

\section{Datos}

En este trabajo se utiliza como fuente de datos la Encuesta Permanente de Hogares 2009 (EPH2009) de la Dirección General de Estadística, Encuestas y Censos (DGEEC), realizada por la Secretaría Técnica de Planificación del Desarrollo Económico y Social de la Presidencia de la República del Paraguay. El objetivo principal de esta encuesta es generar datos relacionados con el empleo, el desempleo, los ingresos y otras características sociales y económicas que permitan conocer la evolución del bienestar de la población paraguaya. La encuesta cubre todos los departamentos del país, excluyendo Alto Paraguay y Boquerón, que representan menos del 2\% de la población total. El tamaño de la muestra es de 4.439 hogares y 18.419 individuos. El levantamiento de los datos se realizó entre octubre y diciembre de 2009. El marco utilizado para la selección de la muestra estuvo constituido por “segmentos", conformados a partir del Censo Nacional de Población y Viviendas 2002. En el ámbito urbano, los segmentos utilizados como unidades primarias de muestreo (UPM) fueron el conjunto de áreas de empadronamiento contiguas hasta alcanzar un promedio de 30 viviendas; en las áreas rurales, en tanto, las UPM se crearon también a partir de las áreas de empadronamiento, que contienen en promedio 35 viviendas.

Las variables de estratificación consideradas fueron el departamento geográfico y el área de residencia. Para la EPH2009 se establecieron 31 estratos, que comprendían a Asunción más los 15 departamentos, divididos cada uno en estratos urbanos y rurales. Por lo tanto, la encuesta está orientada a ser representativa de todo el país, por área urbano-rural de residencia y para los departamentos de San Pedro, Caaguazú, Itapúa, Alto Paraná y Central, más Asunción.

La encuesta posee un módulo sobre empleo e ingreso laboral que indaga sobre la composición de los ingresos mensuales que reciben los miembros del hogar mayores de 10 años. El ítem sobre remesas internacionales corresponde a la categoría "ayuda familiar del exterior" de algún miembro del hogar. Además, se relevan los ingresos provenientes de la ocupación principal, de la ocupación secundaria y de otras ocupaciones (si las hubiera), ingresos por alquileres o rentas, por jubilaciones o pensiones, transferencias familiares regulares en dinero provenientes del país, prestaciones por divorcios y otros ingresos.

Los indicadores de pobreza utilizados son los construidos por la DGEEC (2009b), que estima la proporción de población en situación de pobreza mediante la aplicación del método de la Línea de Pobreza a los datos provenientes de las encuestas de hogares. En este sentido, se define como población pobre a aquel conjunto de personas residentes en hogares cuyo nivel de bienestar (medido a través del ingreso) es inferior al costo de una canasta básica de 
consumo, constituida por el conjunto de bienes y servicios que satisfacen ciertos requerimientos mínimos, tanto alimentarios como no alimentarios, para la sobrevivencia humana.

La Línea de Pobreza se construye estimando primero el costo de una canasta básica de alimentos, cuyo contenido calórico y proteico satisfaga los requerimientos nutricionales, para luego añadirle el costo de la canasta básica no alimentaria, compuesta por otros bienes y servicios esenciales relacionados con la vivienda, la vestimenta, la educación, entre otros aspectos. El costo mensual por persona de la canasta de alimentos se denomina Línea de Pobreza Extrema (LPE), y el de la canasta total recibe el nombre de Línea de Pobreza Total (LPT).

Como puede observarse en la Tabla 2, en 2009 el 35,1\% de la población de Paraguay era pobre y el 18,8\% se encontraba en una situación de pobreza extrema. Estas proporciones eran aún mayores en las áreas rurales, donde casi el 50\% de la población se encontraba en situación de pobreza y el 32,4\% en condiciones de pobreza extrema. Estos datos ilustran las disparidades entre los espacios rurales y los urbanos. De hecho, los menores porcentajes de pobreza y pobreza extrema se registraban en Asunción.

TABLA 2

Incidencia absoluta y relativa de la pobreza, por área y dominio Paraguay - 2009 (1)

\begin{tabular}{|c|c|c|c|c|c|}
\hline \multirow{2}{*}{ Dominio } & \multirow{2}{*}{ Población total } & \multicolumn{2}{|c|}{ Población pobre } & \multicolumn{2}{|c|}{ Población pobre extrema } \\
\hline & & N. & Porcentaje & N. & Porcentaje \\
\hline Total país & 6.244 .981 & 2.191 .857 & 35,1 & 1.175 .331 & 18,8 \\
\hline Área urbana & 3.663 .546 & 905.252 & 24,7 & 340.121 & 9,3 \\
\hline Asunción & 505.538 & 106.470 & 21,1 & 44.236 & 8,8 \\
\hline Central urbano & 1.722 .170 & 471.525 & 27,4 & 134.092 & 7,8 \\
\hline Resto urbano & 1.435 .838 & 327.257 & 22,8 & 161.793 & 11,3 \\
\hline Área rural & 2.581 .435 & 1.286 .605 & 49,8 & 835.210 & 32,4 \\
\hline
\end{tabular}

Fuente: Encuesta Permanente de Hogares, 2009 (DGEEC, 2009a).

(1) Esta tabla utiliza los factores de expansión de la encuesta.

Como se aprecia en la Tabla 3, la comparación directa de los porcentajes de pobreza entre los hogares que reciben remesas del Sur global y aquellos que no lo hacen muestra que la incidencia de la pobreza extrema y no extrema es mayor en los hogares receptores. Asimismo, la proporción de hogares no pobres es menor entre los receptores. A fin de observar solo los efectos de las remesas regionales, las dos categorías que se eligieron fueron los hogares que reciben remesas desde Argentina o Brasil y aquellos que no reciben remesas ni internacionales ni internas.

TABLA 3

Estatus de pobreza de los hogares, según condición perceptora de remesas Paraguay - 2009

\begin{tabular}{|c|c|c|c|c|c|c|}
\hline \multirow{2}{*}{ Estatus de pobreza } & \multicolumn{2}{|c|}{ Remesas del Sur global } & \multicolumn{4}{|c|}{ No recibe remesas } \\
\hline & N. & Porcentaje & N. & Porcentaje & $x^{2}$ & $\mathrm{p}$ \\
\hline Pobreza extrema & 55 & 20,0 & 571 & 18,0 & 145.201 & 0.001 \\
\hline Pobreza no extrema & 57 & 21,0 & 423 & 13,0 & & \\
\hline No pobre & 165 & 60,0 & 2230 & 69,0 & & \\
\hline
\end{tabular}

Fuente: Encuesta Permanente de Hogares, 2009 (DGEEC, 2009a). 


\section{Metodología: efectos de las remesas en la pobreza}

Como señalan diversos autores (ADAMS, 2011; MCKENZIE; SASIN, 2007; YANG; MARTÍNEZ, 2006), hay al menos cuatro complicaciones metodológicas para evaluar el impacto de las remesas: simultaneidad, causalidad inversa, sesgo de selección y sesgo por variables omitidas.

La primera se refiere a que muchas decisiones sobre la migración internacional, las remesas o la educación se toman simultáneamente, por lo que la posibilidad de establecer una secuencia cronológica que permita pensar en relaciones de causalidad potenciales es incierta. La causalidad inversa entre las remesas y la pobreza se presenta porque las remesas pueden reducir la pobreza, pero al mismo tiempo los niveles de pobreza pueden influir en el monto de las remesas recibidas; nuevamente en este caso la posibilidad de establecer un antecedente y un consecuente es dudosa. El sesgo de selección se refiere a la selectividad de las personas que tienden a migrar y a recibir remesas. Por ejemplo, si los hogares con mayores niveles educativos o ingresos tienen más probabilidad de recibir remesas, es imposible identificar el efecto de estas simplemente comparando las características de los hogares con y sin migrantes. Finalmente, cuando la recepción de remesas está influida por características inobservables surge el problema de las variables omitidas, es decir, aquellas que no son captadas por el instrumento que se usa para observar el fenómeno, que pueden influir sobre la recepción de remesas y sobre la pobreza al mismo tiempo.

Existen diversas metodologías para hacer frente a estos problemas. Aquí se utiliza la del "análisis de la puntuación de la propensión" (propensity score analysis) (GUO; FRASER, 2010; HO et al., 2007, 2011; ROSENBAUM; RUBIN, 1983, 1985) a fin de construir grupos de comparación que asemejen las condiciones de un diseño experimental, en el que sea posible la atribución de causalidad. Se generan así dos grupos entre los que comparar: el grupo 1, que recibe remesas, y el grupo 2, que no las recibe pero que es similar al otro en un conjunto de variables de control previamente seleccionadas. El foco de esta metodología consiste en estimar cuáles habrían sido el ingreso de los hogares y las tasas de pobreza si no se hubiesen recibido remesas. El procedimiento se realiza mediante el paquete Matchlt (HO et al., 2007, 2011) del software R (THE R CORE TEAM, 2014).

El método de análisis de la puntuación de la propensión es un procedimiento estadístico relativamente nuevo e innovador que ha probado ser útil en la evaluación de los efectos de algún "tratamiento" cuando se utilizan datos no experimentales, es decir, que provienen de observaciones no controladas (THOEMMES; KIM, 2011). Su aplicación involucra tres pasos (GUO; BARTH; GIBBONS, 2006; GUO; FRASER, 2010).

El primero es la estimación de la puntuación de la propensión, que requiere que se identifiquen las variables cuyos efectos serán bloqueados en la comparación. El segundo es el apareo (matching) de los hogares tratados con los no tratados en función de su puntuación en la propensión, cuyo resultado es una sub-muestra de la original, por lo que este paso se llama re-muestreo. De este modo, los hogares que reciben remesas son pareados con otros 
similares, excepto por el hecho que no las reciben. La literatura reconoce dos algoritmos para hacer el pareo: "el vecino más cercano" y "el vecino más cercano dentro de un calibrador" (GUO; BARTH; GIBBONS, 2006; GUO; FRASER, 2010). El tercer paso es el análisis de los efectos del tratamiento basado en las muestras.

En el presente estudio se estima la probabilidad de recibir remesas desde Argentina o Brasil en hogares paraguayos como función de características observables del hogar y del jefe del hogar, luego se establece un ranking de hogares receptores y no receptores en función de su puntuación en la propensión. A continuación se realiza un apareo entre hogares receptores y no receptores según sus puntuaciones similares en la propensión. Con estos grupos de comparación se calculan las diferencias en los niveles de pobreza. Dado que se analiza el efecto de las remesas provenientes del Sur, no se consideran los hogares receptores de remesas de otros países (en particular Estados Unidos y España) ni del interior del país. El objetivo es trabajar específicamente con el efecto de las remesas regionales en Paraguay.

\section{Paso 1: Estimación de la puntuación de la propensión}

Rosenbaum y Rubin (1985) definen la puntuación de la propensión para los sujetos i $(i=1, \ldots . ., N)$ como la probabilidad condicional de ser asignado a un particular tratamiento $\left(\mathrm{W}_{\mathrm{i}}=1\right)$ versus el no tratamiento $\left(\mathrm{W}_{\mathrm{i}}=0\right)$ dado un vector de covariables observadas $\mathrm{x}_{\mathrm{i}}$ :

$$
e\left(x_{i}\right)=\operatorname{pr}\left(W_{i}=1 / X_{i}=x_{i}\right)
$$

Generalmente este valor es estimado mediante una regresión logística. Rosenbaum y Rubin (1985) sugieren usar el logit de la probabilidad predicha como puntuación de la propensión $(e j: q(x)=\log [(1-\hat{e}(x)) / \hat{e}(x)])$, porque la distribución de $q(x)$ aproxima una distribución normal².

Las principales variables incluidas en el modelo de regresión logística se muestran en la Tabla 4, en la que se presentan los datos estadísticos descriptivos y el análisis bivariado. De los 3.501 hogares paraguayos que componen el universo muestral de la EPH2009, el 7,9\% recibe remesas provenientes del Sur global (Argentina y Brasil). El análisis bivariado (chi cuadrado y pruebas $t$ ) muestra que la mayoría de las variables acusan diferencias estadísticamente significativas entre los dos grupos antes del matching, que indican que la distribución de las covariables no está lo suficientemente superpuesta entre el grupo de control y el grupo bajo tratamiento en la muestra original. El conjunto de variables utilizadas toma en cuenta los antecedentes de investigaciones que utilizaron el mismo enfoque (CLÉMENT, 2011; ESQUIVEL; HUERTA-PINEDA, 2007; JIMÉNEZ-SOTO; BROWN, 2012; LÓPEZ-VIDELA; MACHUCA, 2014; SHARMA, 2011), además de razones teóricas que llevan a determinar las variables que se desea

\footnotetext{
2 En la literatura sobre este tema (ROSENBAUM; RUBIN, 1985; GUO; BARTH; GIBBONS, 2006; GUO; FRASER, 2010), la cantidad $q(x)$ es también denominada puntuación de la propensión, aunque $q(x)$ difiera de $\hat{e}(x)$ en función de la ecuación precedente. En este trabajo se sigue la convención de referir $\hat{e}(x)$ como la puntuación de la propensión. El lector debe tener en consideración que se puede usar una transformación logit de $\hat{e}(x)$, ya que $q(x)$ tiene propiedades que la hacen más manejable que $\hat{e}(\mathrm{x})$.
} 
"bloquear" 3. Se construye así un grupo de comparación constituido por hogares muy similares a los receptores pero que de hecho no reciben remesas ni de Argentina ni de Brasil. Esta primera descripción muestra las principales características de los hogares receptores (véase la Tabla 4):

- mayor proporción de jefatura de hogar femenina;

- mayor edad promedio del jefe de hogar (54,4 años en los receptores y 46,5 años en los otros), diferencia que es estadísticamente significativa;

- mayor proporción de hogares receptores en los que el estado civil del jefe es sin unión; ${ }^{4}$

- menor cantidad de años promedio de escolarización de los jefes de los hogares receptores $(4,5)$ que los no receptores $(7,6)$;

- el idioma principal que hablan los jefes de hogar que reciben remesas es el guaraní;

- menor proporción de personas en edades económicamente activas (18 a 64 años) en los hogares receptores;

- mayor proporción de personas mayores de 64 años en los hogares receptores;

- tipo de hogar extendido como rasgo característico de los receptores;

- mayor proporción de hogares en las áreas rurales;

- mayor tamaño de los hogares receptores (4,5 personas) que de los no receptores (4,2 personas).

\section{Paso 2: Apareo o remuestreo}

Después de obtener las puntuaciones de la propensión estimadas en función de las variables utilizadas en el modelo de regresión logística, se procede a aparear los casos para crear una nueva muestra de aquellos con similares probabilidades de ser asignados al tratamiento (recibir remesas del Sur). El apareo se realiza según los dos algoritmos más utilizados: "vecino más cercano" (nearest neighbor matching) y "vecino más cercano dentro de calibrador" (nearest neighbor matching within a caliper) (GUO; BARTH; GIBBONS, 2006; GUO; FRASER, 2010).

\section{Vecino más cercano}

$\mathrm{P}_{i}$ y $\mathrm{P}_{j}$ son las puntuaciones de la propensión para los grupos de tratamiento y de control, respectivamente; $\mathrm{I}_{1}$ es el grupo de participantes tratados e $\mathrm{I}_{0}$ el de control. Un "vecindario" $\left(\mathrm{P}_{i}\right)$ contiene los participantes de control $\mathrm{j}\left(\mathrm{j} \in \mathrm{I}_{0}\right)$ como también el apareo de los participantes tratados $\mathrm{i}\left(\mathrm{i} \in \mathrm{I}_{1}\right.$ ), si la diferencia absoluta de las puntuaciones de la propensión es la menor entre todos los pares de puntuaciones de la propensión entre i y j:

$$
\mathrm{C}\left(\mathrm{P}_{i}\right)=\min \left\|\mathrm{P}_{i}-\mathrm{P}_{j}\right\|, \mathrm{j} \in \mathrm{I}_{0}
$$

Después de que se encuentra j para aparear a $\mathrm{i}$, j es removido de $\mathrm{I}_{0}$ sin reemplazo. Si para cada i hay solo un j encontrado que cae dentro de $\mathrm{C}\left(\mathrm{P}_{i}\right)$, entonces el apareo es "vecino

\footnotetext{
${ }^{3}$ Como el procedimiento balancea satisfactoriamente las covariables seleccionadas, no se incluyen interacciones en el modelo de regresión logística. Véase HO et al. (2007, p. 219).

${ }^{4}$ En la variable "estado civil” la categoría “en unión” incluye casado y unido, en tanto que la categoría "sin unión” considera separado, viudo, soltero y divorciado.
} 
más cercano 1 a 1". Si por cada i hay n participantes encontrados que caen dentro de C(Pi), entonces el apareo es 1 a $\mathrm{n}$.

TABLA 4

Estadísticas descriptivas y modelo de regresión logística para predecir la puntuación de la propensión Paraguay - 2009

\begin{tabular}{|c|c|c|c|c|c|c|c|c|c|c|}
\hline \multicolumn{2}{|l|}{ Variables } & N. & Porcentaje & $\begin{array}{c}\text { Porcentaje } \\
\text { remesas } \\
\text { Sur }\end{array}$ & Media & $\begin{array}{c}\text { Desviación } \\
\text { estándar }\end{array}$ & gl & $\begin{array}{c}\text { Bivariado. } \\
\mathrm{x}^{2}, \mathrm{t}\end{array}$ & $\mathrm{p}$ & $\begin{array}{c}\text { Logística. } \\
\text { B }\end{array}$ \\
\hline \multicolumn{11}{|c|}{ Jefe de hogar } \\
\hline \multirow[t]{2}{*}{ Sexo } & (Mujer) & 905 & 25,9 & 40,1 & & & 1 & 32 & 0,000 & $-0,689^{\star \star \star}$ \\
\hline & Hombre & 2.594 & 74,1 & 59,9 & & & & & & \\
\hline \multirow[t]{2}{*}{ Edad } & Sin remesas & & & & 46,5 & 15,0 & 330 & -9 & 0,000 & \\
\hline & Sur global & & & & 54,4 & 14,3 & & & & $0,118^{\star \star \star}$ \\
\hline Cuadrado & Sin remesas & & & & 2385 & 1533 & 3497 & -8 & 0,000 & \\
\hline edad & Sur global & & & & 3166 & 1574 & & & & $-0,001^{\star \star}$ \\
\hline \multirow[t]{2}{*}{ Estado civil } & (Sin unión) & 862 & 24,6 & 35,0 & & & 1 & 17 & 0,000 & $-0,103$ \\
\hline & En unión & 2.637 & 75,4 & 65,0 & & & & & & \\
\hline Años de & Sin remesas & & & & 7,6 & 4,5 & 380 & 15 & ,000 & \\
\hline estudio & Sur global & & & & 4,5 & 3,1 & & & & $-0,032$ \\
\hline Cuadrado & Sin remesas & & & & 77,7 & 81,0 & 498 & 17 & ,000 & \\
\hline $\begin{array}{l}\text { años de } \\
\text { estudio }\end{array}$ & Sur global & & & & 30,5 & 40,1 & & & & $-0,007$ \\
\hline Idioma en & Otros & 1.831 & 52,3 & 30,7 & & & 1 & 56 & 0,000 & \\
\hline el hogar & $\begin{array}{l}\text { (Solo } \\
\text { guaraní) }\end{array}$ & 1.668 & 47,7 & 69,3 & & & & & & 0,289 \\
\hline
\end{tabular}

\begin{tabular}{|c|c|c|c|c|c|c|c|c|c|c|}
\hline \multicolumn{11}{|l|}{ Hogar } \\
\hline Proporción & Sin remesas & & & & ,264 & ,231 & 3497 & -1 & ,146 & \\
\hline$<14$ & Sur global & & & & ,285 & ,227 & & & & $1,16^{\star \star \star}$ \\
\hline Proporción & Sin remesas & & & & ,604 & 267 & 3497 & 5 & ,000 & \\
\hline $18-64$ & Sur global & & & &, 521 & ,266 & & & & \\
\hline Proporción & Sin remesas & & & & ,081 & ,219 & 316 & -2 & ,027 & \\
\hline$>64$ & Sur global & & & & ,114 & ,244 & & & & $-0,64$ \\
\hline Ingreso & No tiene & 2.865 & 81,9 & 84,5 & & & 1 & 1 & 0,242 & \\
\hline secundario & Tiene & 634 & 18,1 & 15,5 & & & & & & \\
\hline Tipo de & Unipersonal & 278 & 7,9 & 8,7 & & & 4 & 111 & 0,000 & \\
\hline \multirow[t]{4}{*}{ hogar } & $\begin{array}{l}\text { Nuclear } \\
\text { completo }\end{array}$ & 1.850 & 52,9 & 23,8 & & & & & & \\
\hline & $\begin{array}{l}\text { Nuclear } \\
\text { incompleto }\end{array}$ & 302 & 8,6 & 15,2 & & & & & & \\
\hline & Extendido & 985 & 28,2 & 49,1 & & & & & & \\
\hline & Compuesto & 84 & 2,4 & 3,3 & & & & & & \\
\hline \multirow[t]{2}{*}{ Área } & (Rural) & 1.550 & 44,3 & 62,1 & & & 1 & 39 & 0,000 & $-0,403^{\star *}$ \\
\hline & Urbana & 1.949 & 55,7 & 37,9 & & & & & & \\
\hline Tamaño & Sin remesas & & & & 4,2 & 2,1 & 312,266 & -2 & 034 & \\
\hline hogar & Sur global & & & & 4,5 & 2,5 & & & & \\
\hline
\end{tabular}

Constante de regresión logística

$5,564^{\star \star \star}$

Fuente: Encuesta Permanente de Hogares, 2009 (DGEEC, 2009a).

Nota: Categoría de referencia entre paréntesis. ${ }^{*} p<0,05 ;{ }^{* *} p<0,01 ;{ }^{* * *} p<0,001$. 


\section{Vecino más cercano dentro de un calibrador}

Este método es una combinación de dos abordajes. Se comienza ordenando aleatoriamente los participantes tratados y de control. Se selecciona el primer participante tratado i y después se encuentra j como apareo para i, si la diferencia absoluta de la puntuación de la propensión entre i y j cae dentro de un calibrador $\varepsilon$ determinado, y si el menor de todos los pares de las diferencias absolutas de la puntuación de la propensión entre i y los otros j está dentro del calibrador. Ambos, i y j, son posteriormente removidos de la consideración para el próximo apareo y se selecciona el siguiente participante tratado. El tamaño del calibrador es determinado por el investigador, pero en la literatura se sugiere que sea un cuarto de la desviación estándar de $q(x)$ (ROSENBAUM; RUBIN, 1985), equivalente a $0,25^{\star} \sigma_{q}$. La Tabla 5 presenta el resumen de los esquemas del matching aplicados: el tamaño de las nuevas muestras fue de 277 casos. En el apéndice se expone el análisis gráfico del resultado de ambos esquemas del matching.

\section{TABLA 5}

Esquema del matching y tamaño de las nuevas muestras

Paraguay - 2009

\begin{tabular}{llcc}
\hline \multirow{2}{*}{ Esquema } & \multicolumn{1}{c}{$\begin{array}{c}\text { Descripción del esquema matching y } \\
\text { tamaño de las nuevas muestras }\end{array}$} & \multicolumn{2}{c}{ N. de las nuevas muestras } \\
\cline { 2 - 4 } $\begin{array}{l}\text { Vecino más } \\
\text { cercano }\end{array}$ & $\begin{array}{l}\text { Modelo 1. Puntuación de la propensión basada en } \\
\text { regresión logística, apareo uno a uno a través del vecino } \\
\text { más cercano }\end{array}$ & 277 & Control \\
\hline $\begin{array}{l}\text { Vecino más } \\
\text { cercano dentro } \\
\text { de calibrador }\end{array}$ & $\begin{array}{l}\text { Modelo 2. Puntuación de la propensión basada en } \\
\text { regresión logística, matching uno a uno a través de vecino }\end{array}$ & 277 \\
\hline muente: Encuesta Permanente de Hogares, 2009 (DGEEC, 2009a).
\end{tabular}

Fuente: Encuesta Permanente de Hogares, 2009 (DGEEC, 2009a).

\section{Paso 3: Comparación de las nuevas muestras}

Con la nueva muestra derivada del proceso de apareo (matching) se obtienen grupos comparables o balanceados en relación a la puntuación de la propensión en el tratamiento. El grupo tratado (o experimental) es el compuesto por los hogares que reciben remesas regionales, específicamente desde Argentina o Brasil; el grupo de control (no tratado) es el de los hogares que no reciben remesas desde ningún país de destino de la migración; pero en las variables identificadas en el paso 1 los grupos son homogéneos. La comparación mediante los dos procedimientos produce los resultados que se muestran en la Tabla 6.

Se aprecia que los métodos de apareamiento producen diferencias de poca importancia en el resultado y no modifican la conclusión: la proporción de hogares en situación de pobreza extrema es menor entre los hogares receptores (diferencia estadísticamente significativa). En la misma dirección, la proporción de hogares no pobres es mayor entre ellos. Ambos resultados indican un efecto positivo de la recepción de remesas sobre la pobreza. Sin embargo, esta conclusión debe matizarse, ya que la pobreza no extrema muestra un comportamiento opuesto: afecta en mayor medida a los hogares receptores. 
TABLA 6

Estatus de pobreza de los hogares, según condición perceptora de remesas después del matching Paraguay - 2009

\begin{tabular}{|c|c|c|c|c|c|c|}
\hline \multirow{2}{*}{ Esquemas } & \multicolumn{2}{|c|}{ Remesas del Sur global } & \multicolumn{2}{|c|}{ No recibe remesas } & \multirow[b]{2}{*}{$\mathrm{t}$} & \multirow[b]{2}{*}{$\mathrm{p}$} \\
\hline & Media & $\begin{array}{l}\text { Desviación } \\
\text { estándar }\end{array}$ & Media & $\begin{array}{l}\text { Desviación } \\
\text { estándar }\end{array}$ & & \\
\hline $\begin{array}{l}\text { Esquema 1: vecino ma } \\
\text { cercano dentro de } \\
\text { calibrador } \\
\text { Pobreza extrema }\end{array}$ & 0,199 & 0,400 & 0,329 & 0,471 & 3,5038 & 0,0005 \\
\hline Pobreza no extrema & 0,206 & 0,405 & 0,155 & 0,363 & $-1,5471$ & 0,1224 \\
\hline No pobre & 0,596 & 0,492 & 0,516 & 0,501 & $-1,8838$ & 0,0601 \\
\hline \multicolumn{7}{|c|}{$\begin{array}{l}\text { Esquema 2: vecino más } \\
\text { cercano }\end{array}$} \\
\hline Pobreza extrema & 0,199 & 0,400 & 0,264 & 0,441 & 1,8165 & 0,0349 \\
\hline Pobreza no extrema & 0,206 & 0,405 & 0,202 & 0,402 & $-0,1052$ & 0,9162 \\
\hline No pobre & 0,596 & 0,492 & 0,534 & 0,500 & $-1,4570$ & 0,1457 \\
\hline
\end{tabular}

Fuente: Encuesta Permanente de Hogares, 2009 (DGEEC, 2009a).

\section{Discusión y conclusiones}

El objetivo de este trabajo fue aportar evidencia empírica al análisis de un corredor migratorio Sur-Sur de relevancia para América Latina. En Paraguay, las remesas internacionales revisten una importancia creciente en los últimos años, superando a la inversión extranjera directa. Como se pudo observar, el 12,2\% de los hogares paraguayos recibe este tipo de flujos monetarios, gran parte de los cuales proviene de Argentina. En ausencia de datos experimentales, el estudio del efecto de las remesas internacionales en la pobreza ha sido un campo de extenso debate metodológico. Como no es posible observar los hogares antes y después de haber recibido las remesas, en este artículo se utilizó la técnica de puntuación de la propensión (propensity score analysis). Se emplearon datos de la Dirección General de Estadística, Encuestas y Censos de Paraguay (2009) e indicadores de pobreza construidos por la misma institución. La DGEEC estima la población en situación de pobreza mediante el método de la Línea de Pobreza, considerando una canasta básica de alimentos para la pobreza extrema, a la que luego se añade una canasta no alimentaria de otros bienes esenciales para la pobreza no extrema. Ambos tipos de pobreza tienen mayor incidencia en el área rural $-49,8 \%$ en el caso de la no extrema y 35,4\% en el de la extrema.

Los resultados obtenidos sugieren que en Paraguay las remesas internacionales provenientes del Sur global (Argentina y Brasil) contribuyen a aliviar la situación de pobreza extrema, pero no afectan en la misma magnitud a los hogares en situación de pobreza no extrema.

El resultado coincide en general con los hallazgos de otros autores en cuanto al efecto de la remesas en la reducción de la pobreza en los países receptores, como Adams y Page (2005), Yang; Martínez (2006), Acosta et al. (2008) y Adams (2006a, 2006b, 2011). Pero el matiz sobre los hogares en situación de pobreza no extrema se acerca a lo que encontraron Acosta; Fajnzylber \& López (2007), quienes observaron que el efecto del ingreso proveniente 
de las remesas en la reducción de la pobreza es muy pequeño y que depende del porcentaje de los hogares receptores que pertenecen a los quintiles de ingresos inferiores, en los que los efectos serían mayores. En ese sentido, el presente análisis sobre los datos de Paraguay de 2009 mostraría que los efectos positivos de las remesas son apreciables solo en los niveles más acentuados de la pobreza. Estos resultados son también consistentes con los de Adams y Page (2005), para quienes el incremento de la proporción de migrantes incide negativamente sobre la proporción de población que vive en extrema pobreza.

Hay también concordancia con los hallazgos de Acosta et al. (2008), para quienes las remesas tienen un efecto estadísticamente significativo en la reducción de la pobreza, pero que varía según los países. Alli donde los hogares receptores se concentran en los estratos más bajos de la distribución del ingreso, como Ecuador, El Salvador, Guatemala, México y Paraguay, se advierte una mayor reducción de la pobreza atribuible a las remesas. Aunque el presente estudio no compara países, son los estratos socioeconómicos más desfavorecidos los que acusan el mayor impacto de las remesas.

Con matices en relación a la metodología de medición de la pobreza y la magnitud del efecto, los resultados son convergentes con los estudios que han utilizado la misma metodología (CHUKWUONE et al., 2012; ESQUIVEL; HUERTA-PINEDA, 2007; JIMÉNEZ-SOTO; BROWN, 2012; LÓPEZ-VIDELA; MACHUCA, 2014; SHARMA, 2011).

La migración es un fenómeno multidimensional, y diversos mecanismos contribuyen a explicar el mantenimiento de los flujos de migración regional. En un mercado laboral de alta precarización como el paraguayo, los hogares receptores de remesas regionales se caracterizan por tener condiciones socioeconómicas también precarias (GÓMEZ; BOLOGNA, 2013). Se infiere que las remesas alivian las presiones sobre el mercado laboral, lo que permite que los hogares continúen mandando miembros al exterior a pesar de las precarias y frágiles condiciones de inserción laboral en las que esas remesas se generan. Esta situación se produce en mayor medida en los hogares sometidos a situaciones de pobreza extrema; es allí donde el efecto de los envíos monetarios es mayor.

Ha quedado fuera del alcance de esta comunicación el destino que se da a estos flujos monetarios, que abre interrogantes de importancia sobre su sostenibilidad. Aquellos que no sean capaces de transformarse en inversiones productivas, solo contribuirán a acentuar la dependencia, ya que su uso fundamental será la reproducción de la unidad doméstica. El alivio de la condición de pobreza de los hogares de los estratos socioeconómicos más bajos podría implicar que las remesas desempeñan únicamente un rol de reemplazo de los salarios de los miembros que emigraron.

\section{Referencias}

ACOSTA, P. et al. What is the impact of international remittances on poverty and inequality in Latin America? World Development, v. 36, n. 1, p. 89-114, 2008.

ACOSTA, P.; FAJNZYLBER, P.; LÓPEZ, J. H. The impact of remittances on poverty and human capital: evidence from Latin American household surveys. In: SCHIFF, M.; ÖZDEN, C.. (Eds.). International migration and economic development. Washington, DC: The World Bank and Palgrave Macmillan, 2007, p. 59-98. 
ADAMS, R. Worker remittances and inequality in rural Egypt. Economic Development and Cultural Change, v. 38, n. 1, p. 45-71, 1989.

Remittances, poverty, and investment in Guatemala. In: International migration, remittances, and the brain drain. Washington, DC: World Bank Publications, $2006 \mathrm{a}$.

Remittances and poverty in Ghana. Washington, DC: World Bank, 2006b (World Bank Policy Research Working Paper, 3838). Disponible en: 〈http://www-wds.worldbank.org/servlet/ WDSContentServer/WDSP/IB/2006/01/31/000016406_20060131160228/Rendered/PDF/wps3838. pdf g̀. Acceso en: 4 abr. 2013.

Evaluating the economic impact of international remittances on developing countries using household surveys: a literature review. Journal of Development Studies, v. 47, n. 6, p. 809-828, 2011.

ADAMS, R. H.; PAGE, J. Do international migration and remittances reduce poverty in developing countries? World Development, v. 33, n. 10, p. 1645-1669, 2005.

AYALA, E. Migraciones. Ensayo escrito en Berna en 1913. Santiago de Chile, 1941.

BAKEWELL, O. South-South migration and human development: reflections on African experiences. New York: United Nations Development Programme, 2009 (Human Development Research Papers, 2009/07). Disponible en: 〈http://hdr.undp.org/en/reports/global/hdr2009/papers/HDRP_2009_07. pdf>. Acceso en: 14 mar. 2013.

BARHAM, B.; BOUCHER, S. Migration, remittances, and inequality: estimating the net effects of migration on income distribution. Journal of Development Economics, v. 55, n. 2, p. 307-331, 1998.

BROWNING, H.; GILLESPIE, F. The effect of emigration upon socioeconomic structure: the case of Paraguay. International Migration Review, v. 13, n. 3, p. 502-518, 1979.

BRUNO, S. El proceso migratorio paraguayo hacia Argentina: evolución histórica, dinámica asociativa y caracterización sociodemográfica y laboral. Migrantes paraguayos en Argentina: población, instituciones y discursos. Cuadernos Migratorios. Organización Internacional para las Migraciones (OIM) ed. Buenos Aires, Argentina, 2012, v. 4, p. 11-47.

CANALES, A. I. Remesas y desarrollo en América Latina. Una relación en busca de teoría. Migración y Desarrollo, n. 11, p. 5-30, 2008.

CERRUTTI, M.; PARRADO, E. Remittances of Paraguayan migrants to Argentina: their prevalence, amount and utilization. Integration and Trade Journal, v. 11, p. 21-44, 2007.

CHUKWUONE, N. et al. Analysis of impact of remittance on poverty in Nigeria. [s.l.] Partnership for Economic Policy (PEP), 2012. Disponible en: 〈https://ideas.repec.org/p/lvl/pmmacr/2012-09.htmls. Acceso en: 1 nov. 2014.

CLÉMENT, M. Remittances and household expenditure patterns in Tajikistan: A propensity score matching analysis. Asian Development Review, v. 28, n. 2, p. 58-87, 2011.

COHEN, J. Statistical power analysis for the behavioral sciences. 2. ed. Hillsdale, New Jersey: Routledge, 1988.

DGEEC - Dirección General de Estadística, Encuestas y Censos. Paraguay. Encuesta Permanente de Hogares 2009. Asunción: Dirección General de Estadística, Encuestas y Censos, Secretaría Técnica de Planificación del Desarrollo Económico y Social de la Presidencia de la República, 2009a.

Encuesta Permanente de Hogares de 2009. Principales resultados de Pobreza y Distribución del Ingreso. Asunción: Dirección General de Estadística, Encuestas y Censos, Secretaría Técnica de Planificación del Desarrollo Económico y Social de la Presidencia de la República, 2009b.

ESQUIVEL, G.; HUERTA-PINEDA, A. Remittances and poverty in Mexico: a propensity score matching approach. Integration and Trade Journal, v. 27, 2007. 
GÓMEZ, P. S. Remesas y estratificación social en Paraguay: dinámica de la recepción de remesas del Sur y del Norte. Migraciones, n. 34, p. 77-110, 2013.

. Abordaje contrafáctico, inferencia causal y el enfoque de la puntuación de la propensión. Efectos de las remesas en la escolarización Paraguaya. Empiria. Revista de Metodología de Ciencias Sociales, n. 28, p. 107-128, 2014.

Remesas internacionales, conexiones globales y desarrollo en Paraguay. Estudios Demográficos y Urbanos, en prensa.

GÓMEZ, P. S.; BOLOGNA, E. Remesas y participación laboral en Paraguay. Efectos de los desplazamientos Sur-Sur. Migraciones Internacionales, v. 25, n. 2, p. 189-218, 2013.

GUO, S.; BARTH, R. P.; GIBBONS, C. Propensity score matching strategies for evaluating substance abuse services for child welfare clients. Children and Youth Services Review, v. 28, n. 4, p. 357-383, 2006.

GUO, S. Y.; FRASER, M. W. Propensity score analysis: statistical methods and applications. 1. ed. Thousand Oaks, Estados Unidos: Sage Publications, Inc, 2010.

HECKMAN, J. J. Sample selection bias as a specification error. Econometrica, v. 47, n. 1, p. 153, 1979.

$\mathrm{HO}$, D. et al. Matching as nonparametric preprocessing for reducing model dependence in parametric causal inference. Political Analysis, v. 15, n. 3, p. 199-236, 2007.

Matchit: nonparametric preprocessing for parametric causal inference. Journal of Statistical Software, v. 42, n. 8, p. 1-28, 2011.

HOBBS, A. W.; JAMESON, K. P. Measuring the effect of bi-directional migration remittances on poverty and inequality in Nicaragua. Applied Economics, v. 44, n. 19, p. 2451-2460, 2012.

INDEC - Instituto Nacional de Estadísticas y Censos. Censo nacional de población, hogares y viviendas 2010. Censo del Bicentenario: resultados definitivos. Argentina: INDEC, 2012.

ITZIGSOHN, J. Migrant remittances, labor markets, and household strategies: a comparative analysis of low-income household strategies in the Caribbean Basin. Social Forces, v. 74, n. 2, p. 633-655, 1995.

JIMÉNEZ-SOTO, E. V.; BROWN, R. P. C. Assessing the poverty impacts of migrants' remittances using propensity score matching: the case of Tonga. Economic Record, v. 88, n. 282, p. 425-439, 2012.

LÓPEZ-VIDELA, B.; MACHUCA, C. E. The effects of remittances on poverty at the household level in Bolivia: a propensity score matching approach. Políticas Públicas, v. 2, n. 1, p. 7-22, 2014.

MAGUID, A.; BRUNO, S. Migración, mercado de trabajo y movilidad ocupacional: el caso de los bolivianos y paraguayos en el Área Metropolitana de Buenos Aires. Población de Buenos Aires, v. 7, n. 12 , p. 7-28, 2010.

MÁRQUEZ, C. H. Desarrollo y migración. Una lectura desde la economía política crítica. Migración y Desarrollo, v. 14, p. 59-87, 2010.

MARSHALL, A.; ORLANSKY, D. Inmigración de países limítrofes y demanda de mano de obra en la Argentina, 1940-1980. Desarrollo Económico, v. 23, n. 89, p. 35-58, 1983.

MASSEY, D.; ALARCON, R.; DURAND, J. Return to Aztlan: the social process of international migration from western Mexico (Studies in Demography). Berkeley and Los Angeles, California: University of California Press, 1990.

MCKENZIE, D.; SASIN, M. J. Migration, remittances, poverty, and human capital: conceptual and empirical challenges. Washington, DC: World Bank, 2007 (World Bank Policy Research Working Paper, n. 4272). Disponible en: 〈http://elibrary.worldbank.org/content/workingpaper/10.1596/1813-9450-4272〉. Acceso en: 20 ene. 2013.

OIM - Organización Internacional para las Migraciones. Perfil migratorio de Paraguay 2011. Buenos Aires, Argentina, 2011. 
PALAU, T.; FISCHER, S.; PEREZ, N. Inmigración y emigración en el Paraguay 1870 - 1960. Documento de trabajo. BASE Investigaciones Sociales, Asunción, 1997. Disponible en: <http://biblioteca.clacso.edu. ar/Paraguay/base-is/20120911120450/Doc90.pdf>. Acceso en: 10 feb. 2013.

PARRADO, E. A.; CERRUTTI, M. Labor migration between developing countries: the case of Paraguay and Argentina. International Migration Review, v. 37, n. 1, p. 101-132, 2003.

RATHA, D.; SHAW, W. South-South migration and remittances. Washington, DC: World Bank Publications, 2007.

RIVAROLA, D. Paraguay: estructura agraria y migraciones desde una perspectiva histórica. Migración y desarrollo. Estructura agraria, desarrollo regional, migraciones entre áreas rurales y entre países limítrofes. México: CLACSO, Grupo de Trabajo sobre Migraciones, 1980, p. 1-53.

ROSENBAUM, P. R.; RUBIN, D. B. The central role of the propensity score in observational studies for causal effects. Biometrika, v. 70, n. 1, p. 41-55, 1983.

. Constructing a control group using multivariate matched sampling methods that incorporate the propensity score. The American Statistician, v. 39, n. 1, p. 33-38, 1985.

SHARMA, M. P. International contract-based migration, remittances, and household well-being in the western province of Sri Lanka. International Migration, v. 51, Supplement, p. 1-33, 2011.

STARK, O.; TAYLOR, J. E.; YITZHAKI, S. Remittances and inequality. The Economic Journal, v. 96, n. 383, p. $722-740,1986$

TAYLOR, J. E. The new economics of labour migration and the role of remittances in the migration process. International Migration, v. 37, n. 1, p. 63-88, 1999.

TAYLOR, J. E.; DYER, G. A. Migration and the sending economy: a disaggregated rural economy-wide analysis. Journal of Development Studies, v. 45, n. 6, p. 966-989, 2009.

TAYLOR, J. E.; MORA, J.; ADAMS, R. H. Remittances, inequality and poverty: evidence from rural Mexico. Davis, CA: Department of Agricultural Economics, University of California, 2005 (Working Paper 05-003). Disponible en: 〈http://escholarship.org/uc/item/9s14452d\#page-2〉. Acceso en: 5 mar. 2013.

THE R CORE TEAM. R: A language and environment for statistical computing. Vienna, Austria: $R$ Foundation for Statistical Computing, 2014. Disponible en: 〈http://www.R-project.org〉.

THOEMMES, F. J.; KIM, E. S. A systematic review of propensity score methods in the social sciences. Multivariate Behavioral Research, v. 46, n. 1, p. 90-118, 2011.

WISE, R. D.; MÁRQUEZ, H. Teoría y práctica de la relación dialéctica entre desarrollo y migración. Migración y Desarrollo, n. 9, p. 5-25, 2007.

WORLD BANK. World development indicators. Washington, DC. Disponible en: <http://data.worldbank. org〉. Acceso en: 4 jun. 2013.

YANG, D. C.; MARTÍNEZ, C. Remittances and poverty in migrants' home areas: evidence from the Philippines. In: SCHIFF, M.; ÖZDEN, Ç. (Eds.). International migration, remittances, and the brain drain. Washington, DC: World Bank Publications, 2006, p. 53-80.

\section{Sobre los autores}

Pablo Sebastián Gómez es doctor en Demografía por la Universidad Nacional de Córdoba. Becario postdoctoral, Consejo Nacional de Investigaciones Científicas y Técnicas (CONICET).

Eduardo Bologna es doctor en Demografía por la Universidad Nacional de Córdoba. Profesor-Investigador en el Centro de Estudios Avanzados, Universidad Nacional de Córdoba. 


\title{
Dirección para correspondencia
}

Pablo Sebastián Gómez

Centro de Investigaciones y Estudios sobre Cultura y Sociedad (CIECS)

Universidad Nacional de Córdoba

Rondeau 467 piso 1

Código postal X5000AVI. Córdoba, Argentina

\section{Resumo}

Pobreza e remessas internacionais Sul-Sul no Paraguai

A discussão entre migração e desenvolvimento está fortemente vinculada ao papel das remessas internacionais. Contudo, não existe consenso sobre os efeitos que tais remessas provocam nas economias de origem e nos domicílios receptores. Este artigo analisa o efeito da recepção de remessas regionais no nível e incidência da pobreza em domicílios paraguaios. Para tanto, utilizam-se microdados da Pesquisa Permanente Domiciliar do Paraguai de 2009, da Dirección General de Estadística, Encuestas y Censos (DGEEC, Paraguai). Consideram-se remessas regionais aquelas provenientes da Argentina e do Brasil. Utiliza-se o método de análise da pontuação da propensão (propensity score analysis) para construir grupos comparáveis e, desta forma, isolar o efeito da recepção de remessas de outras variáveis que incidem sobre a condição de pobreza dos domicílios. Os resultados sugerem que as remessas teriam um efeito positivo em aliviar a pobreza extrema e a incidência da pobreza. No entanto, o resultado não é completamente conclusivo, pois a pobreza não extrema afeta em maior medida os domicílios receptores de remessas.

Palavras-chave: Remessas. Pobreza. Inferência casual. Paraguai.

\begin{abstract}
Poverty and south-south financial remittances in Paraguay

There is a debate going on about international financial remittances in terms of the connections between migration and development. However, there is no consensus as to the effects that these remittances have on economies of origin and on receiving households. In this paper we analyze the effect of the receiving of regional remittances on the level and incidence of poverty among Paraguayan households. We use micro data from the 2009 Permanent Household Survey, conducted by the General Department of Statistics, Surveys and Censuses (DGEEC, Paraguay). Regional remittances are defined as those sent from Argentina and Brazil. We applied the method of propensity score analysis to construct comparable groups and thus isolate the effect of reception of regional remittances from other variables that affect the poverty status of households. Results suggest that remittances do have a positive effect in alleviating extreme poverty and the incidence of poverty. However, the result is not entirely conclusive, since nonextreme poverty more strongly affects the households that receive remittances.
\end{abstract}

Keywords: Remittances. Poverty. Causal inference. Paraguay.

\section{Apéndice}

Para Thoemmes y Kim (2011) existe un debate en el campo de la metodología sobre la mejor manera de evaluar el apareo. Autores como Ho et al. (2007) critican las pruebas de significación debido a la dependencia del tamaño de la muestra, y sugieren examinar las diferencias estandarizadas antes y 
después del apareo. Esta diferencia estandarizada es una medida muy utilizada en el campo de la psicología, conocida como "d de Cohen" (COHEN, 1988). El argumento que sustenta esta propuesta es que el uso de las pruebas de significación -como la prueba t- puede ser erróneo en algunas circunstancias debido a una baja potencia estadística. El desarrollo de la metodología puede encontrarse en Gómez (2014). En los Gráficos 1 y 2 se muestran las diferencias de las medias estandarizadas (d de Cohen) antes y después del matching.

GRÁFICO 1

Diferencias de medias estandarizadas (d de Cohen), vecino más cercano dentro de calibrador

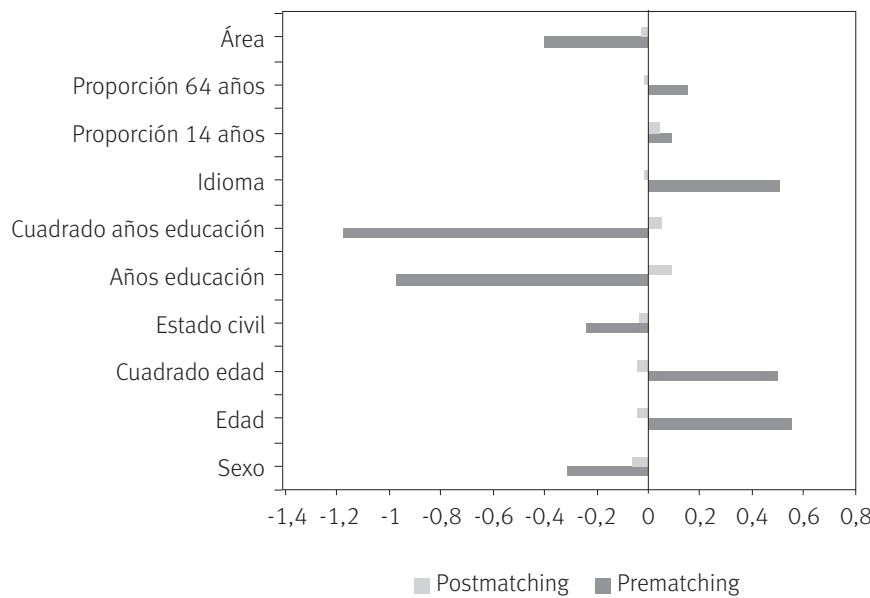

Fuente: Encuesta Permanente de Hogares, 2009 (DGEEC, 2009a).

GRÁFICO 2

Diferencias de medias estandarizadas (d de Cohen), vecino más cercano

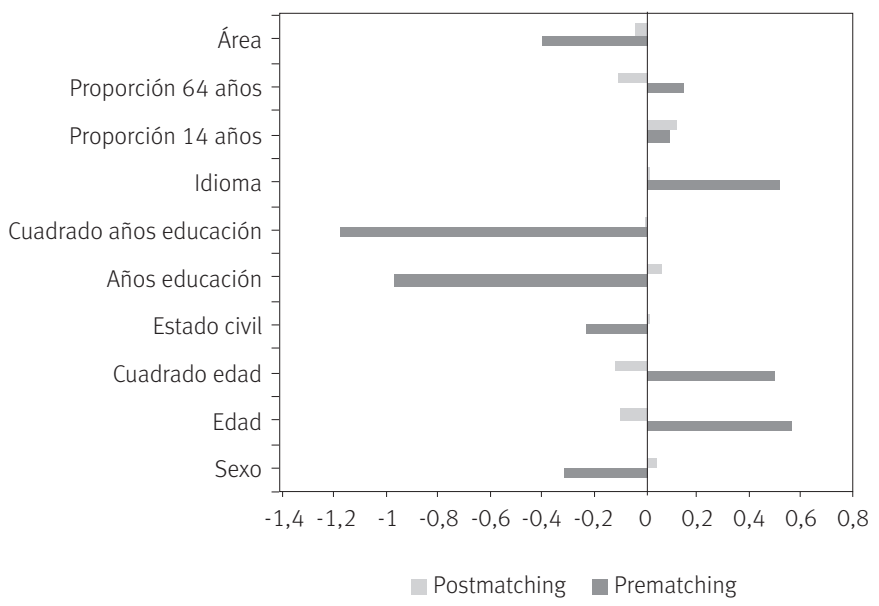

Fuente: Encuesta Permanente de Hogares, 2009 (DGEEC, 2009a).

Recebido para publicação em 31/07/2013

Aceito para publicação em 05/11/2014 
